Mauro Batista de Morais 1

Ulysses Fagundes Neto 1

Ângela Peixoto de Mattos 2

Roberto Geraldo Baruzzi 3

\section{Estado nutricional de crianças índias do Alto Xingu em 1980 e 1992 e evolução pondero-estatural entre o primeiro e o quarto anos de vida}

\author{
Nutritional status of indigenous children \\ from the Alto Xingu in 1980 and 1992 and \\ follow-up of weight and height from the first \\ through the fourth years of life
}

1 Departamento de Pediatria, Escola Paulista de Medicina, Universidade Federal de São Paulo. Rua Botucatu 598, São Paulo, SP 04023-062, Brasil. mbmorais.dped@epm.br ulyneto@osite.com.br 2 Departamento de Pediatria, Faculdade de Medicina, Universidade Federal da Bahia. Av. Reitor Miguel Calmon $s / n$, Salvador, $B A$ 40110-100, Brasil. matossap@ufba.br 3 Departamento de Medicina Preventiva, Escola Paulista de Medicina, Universidade Federal de São Paulo. Rua Pedro de Toledo 675, São Paulo, SP 04039-031, Brasil. baruzzi@medprev.epm.br

\begin{abstract}
This study focused on the under-five population of the Alto Xingu region in Brazil, with the following objectives: (1) to evaluate height and weight increment from the first through the fourth years of life and (2) to compare nutritional status in 1980 and 1992. Height and weight increases were evaluated in 81 children. Weight and height were measured in 264 children evaluated in 1980 and in 172 in 1992 (< 10 years of age). Median Z-scores in the first and fourth years of life, respectively, showed: (1) a decrease in weight-for-age, (-0.12 in the first year and -0.51 in the fourth year of life; $p=0.002)$; (2) a decrease in weight-for-height $(+1.31$ and $+0.08 ; p<0.001$ ); (3) an increase in height-for-age (-1.50 and -0.94; $p<0.001)$. Median Z-scores in 1980 and 1992 showed: (1) no change in weight-for-age (-0.61 in 1980 and -0.62 in 1992; $p=$ 0.90); (2) no change in weight-for-height $(+0.27$ and $+0.34 ; p=0.10)$; and (3) a decrease in height-for-age (-1.04 and -1.22; $p=0.02)$. Height-for-age increased and weight-for-height decreased between the first and fourth years of life. A decrease in height-for-age was observed from 1980 to 1992, demonstrating the importance of nutritional surveillance among the population of the Alto Xingu.
\end{abstract}

Key words Child Nutrition; Anthropometry; Child Welfare; South American Indians

Resumo Os objetivos deste estudo realizado com a população infantil do Alto Xingu foram: (1) analisar a evolução do peso e da estatura entre o primeiro e o quarto anos de vida, (2) comparar o estado nutricional em 1980 e 1992. Avaliaram-se o peso e a estatura de: (1) 81 crianças no primeiro e no quarto ano de vida; (2) 264 crianças avaliadas em 1980 e de 172 em 1992 (idade < 10 anos). As medianas dos escores $Z$ das 81 crianças examinadas no primeiro e no quarto ano de vida revelaram: (1) diminuição do peso para a idade (-0,12 no primeiro ano e -0,51 no quarto ano de vida; $p=0$,002); (2) diminuição do peso para a estatura (+1,31 e+0,08; $p<0,001)$; (3) aumento da estatura para a idade (-1,50 e-0,94, $p<0,001)$. Entre 1980 e 1992, observou-se: (1) manutenção do peso para a idade (-0,61 em 1980 e-0,62 em 1992; $p=0,90)$; (2) manutenção do peso para a estatura, $(+0,27 e+0,34 ; p=0,10)$; $e$ (3) redução da estatura para a idade $(-1,04 e-1,22 ; p=$ 0,02). Entre o primeiro e quarto ano de vida observou-se redução do déficit de estatura para a idade e do excesso de peso para a estatura. Entre 1980 e 1992, observou-se diminuição da estatura para idade, indicando a necessidade de monitorização do estado nutricional desta comunidade. Palavras-chave Nutrição da Criança; Antropometria; Saúde da Criança; Índios Sul-Americanos 


\section{Introdução}

O estado nutricional de crianças de grupos indígenas brasileiros foi avaliado em alguns estudos publicados ao longo das últimas décadas (Alves et al., 2002; Black et al., 1977; Fagundes Neto et al., 1981; Gugelmin et al. 2001; Martins \& Menezes, 1994; Mattos et al., 1999; Morais et al., 1990; Santos \& Coimbra Jr., 1991). Nos estudos nos quais peso e estatura foram relacionados com a idade, observou-se ocorrência de déficit da estatura para a idade em proporção expressiva das crianças estudadas e adequação do peso para a estatura (Alves et al., 2002; Gugelmin et al., 2001; Martins \& Menezes, 1994; Mattos et al., 1999; Santos \& Coimbra Jr., 1991). Este padrão antropométrico pode ter duas explicações não excludentes: (1) déficit de estatura como conseqüência de desnutrição pregressa (Waterlow, 1972), responsável por comprometimento irreversível do crescimento; e/ou (2) déficit de estatura refletindo o potencial genético destes grupos populacionais que, mesmo sem terem apresentado desnutrição no passado, serão constituídos por adultos com estatura média inferior à dos povos dos países do mundo ocidental contemporâneo. Deve-se ressaltar que nos estudos de Fagundes Neto et al. (1981), Morais et al. (1990), Santos \& Coimbra Jr. (1991), Martins \& Menezes (1994), Alves et al. (2002), Mattos et al. (1999) e Gugelmin et al. (2001) com grupos indígenas do Brasil, praticamente a totalidade das crianças avaliadas, independentemente da faixa etária, não apresentava déficit de peso para a estatura.

A validade de se empregar as tabelas de referência de peso e estatura do NCHS (National Center for Health Statistics), adotada pela Organização Mundial da Saúde (OMS) desde 1977 (Waterlow et al., 1977) como referência internacional, na avaliação do crescimento e do estado nutricional de crianças índias brasileiras tem sido por vezes discutida (Gugelmin et al., 2001; Santos, 1993), mostrando a importância da realização de estudos, em nosso país, voltados à avaliação do estado nutricional e crescimento de crianças índias (Campos Jr., 2001).

Entre 1974 e 1980 (Fagundes Neto et al., 1981; Morais et al., 1990) e em 1992 (Mattos et al., 1999) foram realizados inquéritos sobre o estado nutricional de crianças índias do Alto Xingu. No presente artigo são analisadas informações não abordadas nas publicações anteriores (Fagundes Neto et al., 1981; Mattos et al., 1999; Morais et al., 1990). Os objetivos deste estudo foram: (1) analisar a evolução do peso e da estatura das crianças índias do Alto Xingu entre o primeiro e o quarto ano de vida; (2) comparar o estado nutricional de crianças índias do Alto Xingu avaliadas em 1980 e 1992, utilizando índices antropométricos dependentes da idade.

\section{Métodos}

Casuística: inquéritos do estado nutricional de crianças do Alto Xingu

De 1974 a 1980, realizaram-se inquéritos nutricionais anuais (Fagundes Neto et al., 1981; Morais et al., 1990). No primeiro ano, foram incluídas crianças com idade estimada menor do que cinco anos. A cada ano subseqüente, até 1980, procurou-se reexaminar as crianças avaliadas no(s) ano(s) anterior(es) e incluir no estudo tanto as nascidas no transcorrer dos doze meses precedentes, bem como as ausentes em etapas anteriores. Assim, entre 1974 e 1980, cada criança foi examinada no mínimo uma vez e no máximo sete. $\mathrm{O}$ trabalho de campo foi realizado sempre no mês de julho e em todos os anos procurou-se estudar o maior número possível de crianças. Para as crianças nascidas a partir de 1974, contou-se com o registro da data de nascimento, ou quando faltava esta informação, era relativamente fácil avaliar a idade com reduzida margem de erro. Das 94 crianças incluídas no primeiro ano de idade entre 1974 e 1977, 81 (86,2\%) foram reexaminadas no quarto ano de vida. De acordo com informações do cadastro médico da população, uma das crianças foi a óbito no primeiro ano de vida, enquanto as demais não foram examinadas no quarto ano de vida por não estarem presentes no momento da realização do trabalho de campo. Das 81 crianças estudadas no primeiro e no quarto anos de vida, 67 foram avaliadas no segundo ano e 66 no terceiro.

$\mathrm{Na}$ avaliação realizada em 1980, examinaram-se 264 crianças, sendo 28 (10,6\%) com idade inferior a 1 ano, 117 (44,3\%) com idade entre 1 e 5 anos incompletos e 117 (44,3\%)com idade estimada entre 5 e 10 anos. Em setembro de 1992, em novo inquérito nutricional, avaliaram-se 172 crianças: 25 (14,5\%) com idade inferior a um ano, 78 (45,3\%) com idade entre 1 e 5 anos incompletos e $69(40,1 \%)$ com idade entre 5 e 10 anos.

Em suma, a comparação do estado nutricional entre 1980 e 1992, das crianças índias do Alto Xingu com idade menor do que dez anos, baseou-se em índices antropométricos dependentes da idade aplicados para 264 crianças examinadas em 1980 e 172 examinadas em 1992. 
Parque Indígena do Xingu e tribos do Alto Xingu

O Parque Indígena do Xingu (PIX) foi criado em 1961, quando a venda indiscriminada de terras da região central do Brasil, no país e exterior, colocava em risco territórios habitados por tribos indígenas desde tempos imemoráveis. Suas principais finalidades: (1) preservação física e cultural das tribos que ali viviam; (2) acolher tribos de outras áreas geográficas ameaçadas de extinção por choques com as frentes invasoras; (3) preservação da fauna e da flora (Baruzzi et al., 1990).

O PIX ocupa uma área de $32.000 \mathrm{~km}^{2}$, ao norte do Estado de Mato Grosso, que se estende ao longo da calha do Rio Xingu, desde a região de seus formadores, ao sul, denominada Alto Xingu, até a cachoeira de von Martius, ao norte, no limite entre os Estados de Mato Grosso e Pará (Baruzzi et al., 1990).

A região do Alto Xingu é habitada por dez tribos indígenas que apresentam padrões culturais muito semelhantes, denominado de cultura do Alto Xingu ou do Uluri (pequeno cinto pubiano usado pelas mulheres). A acentuada similaridade cultural deve ser resultado da longa ocupação de uma mesma área geográfica e da freqüência de casamentos intertribais. São as seguintes as tribo do Alto Xingu, segundo o tronco ou família lingüística: Aruák (Mehináku, Yawalapití e Waurá), Karib (Kalapálo, Kuikúru, Matipú, e Nahukwá), Tupi (Awetí e Kamayurá) além dos índios Trumái, de língua isolada.

A alimentação dos índios do Alto Xingu é constituída basicamente de mandioca e peixe. Outros alimentos são consumidos com menor regularidade, tais como banana, milho, batata doce e amendoim. Em certas épocas do ano, o piqui, fruto óleoginoso rico em vitamina $\mathrm{A}, \mathrm{e}$ ovos e carne de tracajá (Fagundes Neto et al., 1981; Morais et al., 1990) são bastante consumidos pela população. Por tabus alimentares, os índios não consomem a carne de caça, com exceção para algumas espécies de macacos e aves de grande porte, como o mutum e jaó. O aleitamento materno se estende até os dois ou três anos da criança. No primeiro semestre de vida, parte das crianças recebe também mingau de mandioca. Já no segundo semestre são introduzidos o beiju de mandioca e o peixe (Mattos et al., 1999).

A partir de 1965, a Universidade Federal de São Paulo/Escola Paulista de Medicina desenvolve um programa de atenção à saúde dos índios do PIX, coordenado pelo Departamento de Medicina Preventiva (Baruzzi et al., 1990). Concomitantemente às ações de saúde e imu- nização, desenvolvem-se projetos de pesquisa, entre os quais se inserem inquéritos nutricionais. A adoção de uma ficha médica individual desde os primórdios do programa permite a identificação pessoal por família e etnia, o registro das vacinas aplicadas e das ocorrências clínicas (Baruzzi et al., 1990).

\section{Método}

Mensuraram-se o peso e a estatura de acordo com as recomendações de Jelliffe (1968) para os estudos comunitários de avaliação nutricional. O peso foi aferido com as crianças despidas. Utilizaram-se balanças da marca Filizola, mecânica entre 1974 e 1980 e microeletrônica em 1992. A estatura dos menores de dois anos foi medida com o auxílio de antropômetro de madeira, estando a criança em decúbito dorsal sobre uma superfície retilínea. As crianças com mais de dois anos foram medidas em pé, sendo utilizada fita métrica inelástica aderida à uma superfície vertical de 1974 a 1980 e em 1992 foi utilizada uma fita da marca Stanley. A balança microeletrônica e a fita inelástica Stanley eram idênticas às utilizadas no levantamento nacional do estado nutricional realizado pelo Instituto Nacional de Alimentação e Nutrição (INAN, 1990) no final da década de 1980.

\section{Índices antropométricos e análise estatística}

A partir das medidas do peso e estatura calcularam-se os escores $\mathrm{Z}$ de peso-idade, peso-estatura e estatura-idade com o emprego do programa Epi Info versão 6.2, com base nos valores de referência da tabela do NCHS (WHO, 1983). Como limite de corte para caracterização de desnutrição, de acordo com os escores Z, foi adotado o valor de -2,0 desvios-padrão conforme recomendado pela OMS (WHO, 1995). A análise estatística foi realizada com o emprego do programa Sigma Stat. Os testes estatísticos utilizados estão especificados na apresentação dos resultados.

\section{Resultados}

Na Tabela 1, apresentam-se a média da idade e os escores $\mathrm{Z}$ do peso para a idade, peso para a estatura e estatura para a idade na avaliação pareada entre o primeiro e o quarto ano de vida de 81 crianças índias incluídas no estudo no primeiro ano de vida entre 1974 e 1977 . Observou-se diminuição estatisticamente significante na mediana dos escores $\mathrm{Z}$ do peso para a idade e do peso para a estatura. Quanto ao es- 
Mediana da idade e dos escores Z no primeiro e no quarto anos de vida de crianças índias do Alto Xingu nascidas entre 1974 e 1977.

\begin{tabular}{lccc}
\hline & $\begin{array}{c}\text { 10 ano de vida (percentil } \\
\text { 25; percentil 75) }\end{array}$ & $\begin{array}{c}\text { 4o ano de vida (percentil } \\
\text { 25; percentil 75) }\end{array}$ & Teste de Wilcoxon \\
\hline Idade (meses) & $6,0(4,0 ; 10,0)$ & $42,0(40,0 ; 46,0)$ \\
Escore Z - peso-idade & $-0,12(-0,60 ;+0,42)$ & $-0,51(-0,94 ;+0,21)$ & 0,002 \\
Escore Z - peso-estatura & $+1,31(+0,70 ;+0,20)$ & $+0,08(-0,20 ;+0,77)$ & $<0,001$ \\
Escore Z - estatura-idade & $-1,50(-1,89 ;-0,92)$ & $-0,94(-1,39 ;-0,27)$ & $<0,001$ \\
\hline
\end{tabular}

Parâmetros das crianças avaliadas no segundo e terceiro anos de vida:

Segundo ano $(n=67)$ - idade $=18,0[16,0 ; 21,0]$ meses; $Z$ peso-idade $=-0,77[-1,25 ;-0,14]$;

$Z$ peso-estatura $=+0,47[+0,01 ;+0,94] ; Z$ estatura-idade $=-1,67[-2,13 ;-1,18]$.

Terceiro ano $(n=66)$ - idade $=30,0[28,0 ; 33,0]$ meses; $Z$ peso-idade $=-0,52[-1,08 ;+0,20]$;

$Z$ peso-estatura $=+0,23[-0,22 ;+0,65] ; Z$ estatura-idade $=-0,80[-1,40 ;-0,39]$

core $\mathrm{Z}$ da estatura para a idade, observou-se redução estatisticamente significante no déficit de estatura, em relação aos valores de referência do NCHS. Ainda na Tabela 1, podem-se ver, também, as medianas e percentis 25 e 75 dos escores $\mathrm{Z}$ de peso-idade, peso-estatura e estatura-idade, obtidos no segundo $(n=67)$ e terceiro ano de vida $(n=66)$.

Nas Figuras 1, 2 e 3 pode-se observar a distribuição dos escores $\mathrm{Z}$ do peso para a idade, peso para a estatura e estatura para a idade, nos anos de 1980 e 1992, em relação à distribuição normal esperada para cada um dos indicadores. A análise visual destas três figuras mostra que as curvas de distribuição dos escores Z em 1980 e 1992 são praticamente superpostas. Por sua vez, na análise estatística dos dados correspondentes a estes gráficos, com o emprego do teste de Mann-Whitney, observaram-se os seguintes valores para a mediana (percentis 25 e 75, apresentados entre colchetes) dos escores $\mathrm{Z}$ obtidos em $1980(\mathrm{n}=264)$ e em 1992 ( $n=172)$ e o valor do p na comparação entre os dois anos: peso-idade, -0,61 [-1,09; $+0,03]$ em 1980 e -0,62 [-1,22; +0,11] em 1992 ( $\mathrm{p}=0,90)$; peso-estatura, $+0,27[-0,17 \mathrm{e}+0,72]$ em 1980 e +0,34 [-0,13; +0,91] em $1992(p=0,10)$; e estatura-idade, $-1,04[-1,54 ;-0,56]$ em 1980 e $-1,22[-1,89 ;-0,67]$ em $1992(p=0,02)$. Considerando a diferença estatística observada para o indicador estatura-idade, na Tabela 2 são apresentadas as proporções de crianças com escore $\mathrm{Z}$ inferior a -2,0 desvios-padrão de estatura para a idade, nos anos de 1980 e 1992, de acordo com a faixa etária. Observou-se aumento estatisticamente significante na proporção de crianças com déficit de estatura para a idade, entre os anos de 1980 e 1992, na faixa etária entre 12 e 60 meses. No que se refere à comparação na proporção de déficit de estatura para idade, nas outras faixas de idade, não se observou diferença estatisticamente significante.

\section{Discussão}

Em linhas gerais, a avaliação antropométrica das crianças do Alto Xingu foi concordante com outros estudos realizados em comunidades indígenas brasileiras (Alves et al., 2002; Gugelmin et al., 2001; Martins \& Menezes, 1994; Santos \& Coimbra Jr., 1991), ou seja, presença de déficit de estatura para a idade e adequação do peso para a estatura, sem a caracterização de desnutrição aguda ou atual (Santos, 1993), em relação ao padrão antropométrico de crianças do mundo ocidental de país desenvolvido (Waterlow et al., 1977).

No Alto Xingu, demonstrou-se que, no primeiro ano de vida, as crianças apresentavam peso acima do esperado para estatura e déficit da estatura para a idade em relação à referência do NCHS (Mattos et al., 1999). Desta maneira, a inexistência de déficit de peso no primeiro ano de vida, quando já se observa déficit de estatura, indica que o último não pode ser considerado conseqüência de desnutrição pregressa ("stunting") (Waterlow, 1972). Este aspecto leva a especulação de que o déficit de estatura no primeiro ano de vida possa estar relacionado com as características genéticas da população, expressando-se desde o primeiro ano e contrariando o axioma de que as diferenças no potencial genético para o crescimento tornamse evidentes apenas após o quarto ano de vida (Sigulem et al., 2000). Uma análise mais profunda deste aspecto, ou seja, do déficit de estatura para a idade no primeiro ano de vida, pro- 
Distribuição dos escores Z do peso para a idade das crianças índias do Alto Xingu em 1980 e 1992.

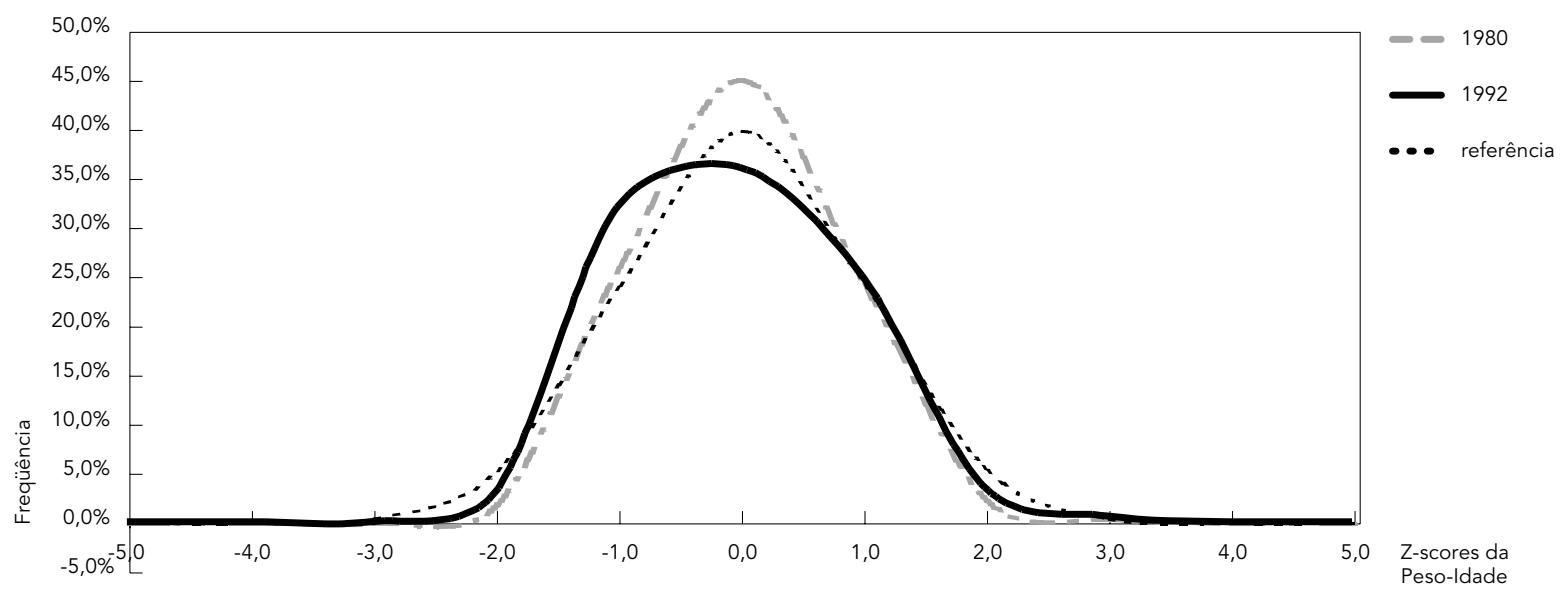

Figura 2

Distribuição dos escores Z do peso para a estatura das crianças índias do Alto Xingu em 1980 e 1992.

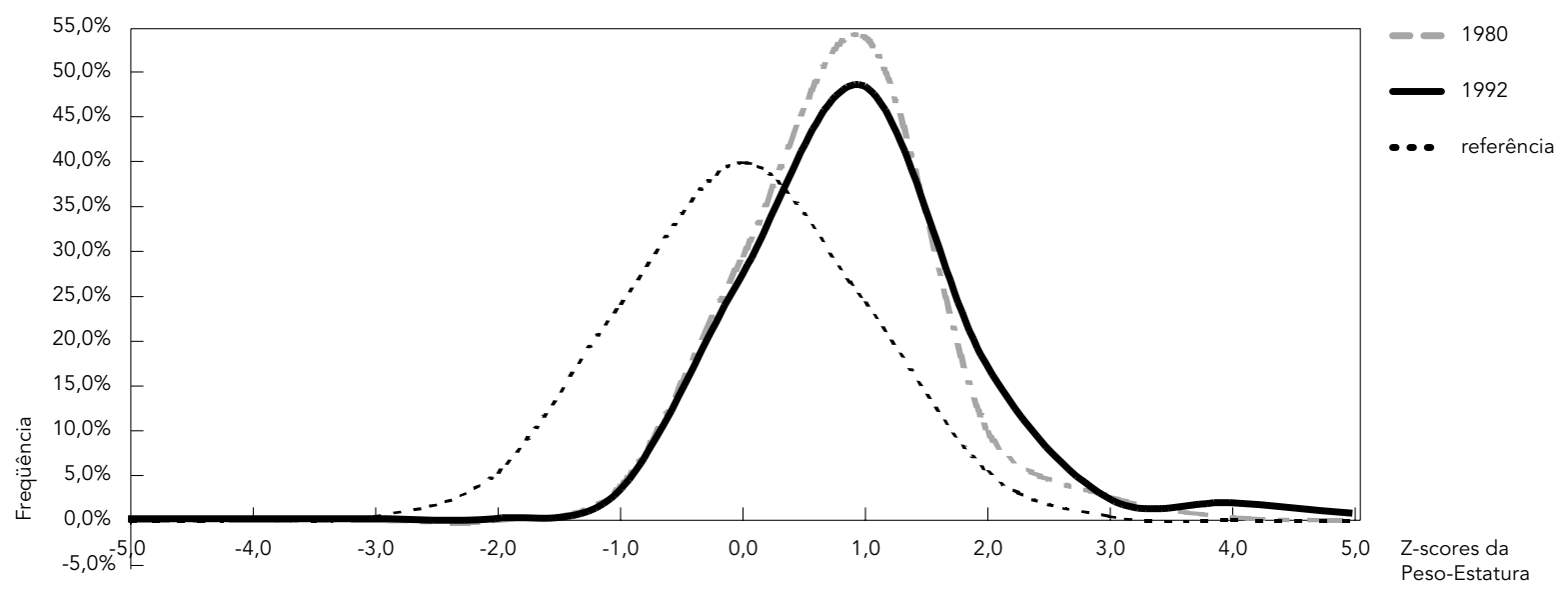

vavelmente não associado com desnutrição primária, foi realizada com o estudo da evolução do peso e da estatura das crianças índias entre o primeiro e o quarto ano de vida. Caso as crianças sofressem de desnutrição crônica, a expectativa seria de diminuição progressiva dos índices dependentes do peso e, também, aumento do déficit de estatura para a idade em função da cronicidade do processo. Pode-se observar, nos resultados da Tabela 1, redução estatisticamente significante no déficit de esta- tura para idade, mostrando indiretamente que as crianças não apresentaram desnutrição crônica entre o primeiro e o quarto ano de vida, o que resultaria no aumento do déficit de estatura para a idade, ao contrário dos resultados obtidos. Esta interpretação contrasta com a opinião de Santos (1993), que, revendo a literatura sobre o crescimento físico e o estado nutricional de populações indígenas brasileiras, assinala que, apesar da proporcionalidade corporal demonstrada pela adequação do peso para 
Figura 3

Distribuição dos escores Z da estatura para a idade das crianças índias do Alto Xingu em 1980 e 1992.

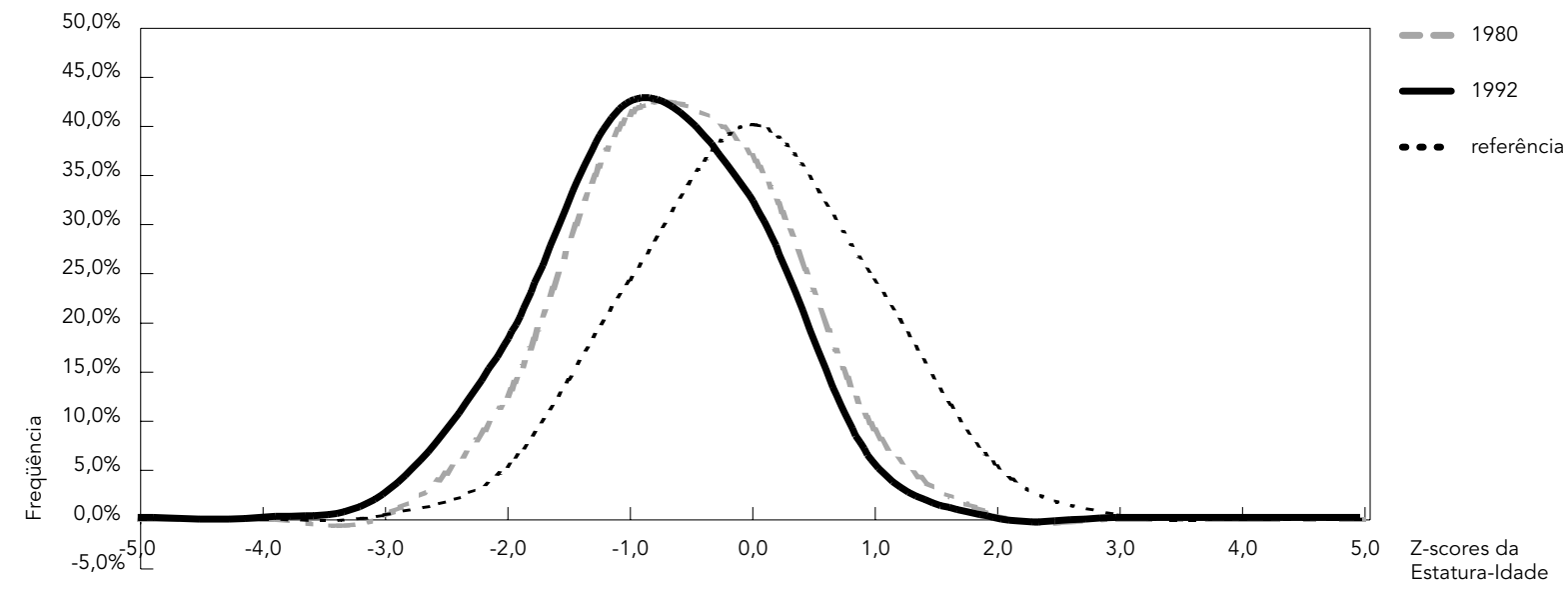

Tabela 2

Déficit de estatura para a idade (escore $Z<-2,0$ desvios-padrão) nas crianças índias do Alto Xingu nos anos de 1980 e 1992.

\begin{tabular}{|c|c|c|c|c|c|}
\hline \multirow{2}{*}{$\begin{array}{l}\text { Faixa etária } \\
\text { (meses) }\end{array}$} & \multicolumn{2}{|c|}{1980} & \multicolumn{2}{|c|}{1992} & \multirow{2}{*}{$\begin{array}{l}\text { Teste do Qui- } \\
\text { Quadrado (p) }\end{array}$} \\
\hline & $Z<-2,0 D P$ & Total & $Z<-2,0 D P$ & Total & \\
\hline $0-12$ & $6(21,4 \%)$ & 28 & $4(16,0 \%)$ & 25 & 0,88 \\
\hline $12-60$ & $12(10,1 \%)$ & 119 & $17(21,8 \%)$ & 78 & 0,04 \\
\hline $60-120$ & $16(13,7 \%)$ & 117 & $13(18,8 \%)$ & 69 & 0,47 \\
\hline
\end{tabular}

Análise estatística: comparação das classes de faixa etária em 1980 e 1992 (0-12 versus 12-60 versus 60-120).

1980: $p=0,26 ; 1992: p=0,79$. a estatura, o déficit de estatura constituiria evidência de altas freqüências de desnutrição energética-protéica crônica ou pregressa. Deve-se salientar, também, que no quarto ano de vida a mediana do escores $Z$ da estatura para a idade permaneceu, em relação à tabela do NCHS, com desvio para a esquerda, sendo a mediana do escore $\mathrm{Z}$ igual a $-0,94$. Vale lembrar que a estatura média dos adultos do sexo masculino $(161,0 \mathrm{~cm})$ e feminino $(151,8 \mathrm{~cm})$ do Alto Xingu (Franco, 1981) é inferior aos valores médios de estatura da tabela do NCHS para os 18 anos de vida.

Nossos resultados mostraram, também, diminuição estatisticamente significante na análise pareada dos escores $\mathrm{Z}$ do peso para a idade e do peso para a estatura. Este aspecto já havia sido observado em estudo anterior (Morais et al., 1990) no qual a elevada proporção de crianças no primeiro ano de vida com adequação percentual do peso para a estatura entre $110 \%$ e $120 \%(26,2 \%$; $51 / 195)$ e maior do que $120 \%$ $(37,4 \% ; 73 / 195)$ no primeiro ano de vida despertou a preocupação de que esta tendência de excesso de peso poderia persistir durante a infância (Morais et al., 1990). No entanto, durante o acompanhamento destes lactentes em anos subseqüentes, observou-se redução na proporção de crianças com aumento da adequação percentual do peso para a estatura (Morais et al., 1990), que no presente estudo, foi caracterizada pela diminuição na mediana do escores $\mathrm{Z}$ do peso para a estatura de $+1,31$ no primeiro ano para $+0,08$ no quarto ano de vida.

A caracterização de déficit de estatura para a idade não associado, ao que tudo indica, com desnutrição no primeiro ano de vida, parcialmente reversível entre o primeiro e quarto ano de vida e o excesso de peso para a estatura no primeiro ano de vida sugerem que a tabela do NCHS não seria plenamente adequada para avaliar o estado nutricional das crianças índias do Alto Xingu. É possível que esta observação possa ser válida também para outros povos indígenas, conforme assinalado por Santos (1993) e Gugelmin et al. (2001). Por sua vez, seria muito difícil elaborar valores de referência específicos para crianças índias, dado que existe grande dispersão dos povos indígenas no território nacional em comunidades muitas vezes de difícil acesso, em geral, com número reduzido de 
habitantes, além da acentuada diversidade sócio-cultural. Deve-se lembrar, ainda, que os valores de referência são medidas que fornecem uma linha de base para comparar diferentes grupos populacionais, ou o mesmo grupo em diferentes momentos, não tendo implicações obrigatórias com o complexo e insolúvel conceito de "normalidade".

Observando-se as Figuras 1, 2 e 3, pode-se constatar que não ocorreu mudança expressiva nos indicadores antropométricos do peso para a idade, peso para a estatura e estatura para a idade no período entre 1980 e 1992, ou seja, não se observou alteração na condição nutricional das crianças índias do Alto Xingu. A comparação das curvas de distribuição dos escores $\mathrm{Z}$ do peso para a idade, peso para a estatura e estatura para a idade, em relação à curva de referência mostra que as distribuições do peso para a idade são muito semelhantes à referência, ao passo que as curvas do peso para a estatura se deslocam para a direita. Por sua vez, as distribuições da estatura para a idade desviamse para a esquerda da referência tanto em 1980 como em 1992. No que se refere aos valores dos escores $\mathrm{Z}$ da estatura para a idade nas crianças índias do Alto Xingu, observou-se que a mediana diminui de -1,04 em 1980 para -1,22 em 1992, sendo esta diferença estatisticamente significante. De acordo com a Tabela 2, a proporção de crianças com escore $\mathrm{Z}$ inferior a -2,0 desvios-padrão, em 1992 em relação a 1980, foi estatisticamente superior na faixa etária entre 1 e 5 anos. Deve-se ressaltar que, nas faixas etárias inferior a 1 ano e entre 5 e 10 anos, não se caracterizou aumento significante na proporção de crianças com estatura para idade abaixo de -2,0 desvios-padrão. Para a faixa etária entre 1 e 5 anos, a diferença entre as medianas de aproximadamente 0,20 desvios-padrão (cerca de 2-3 centímetros segundo a tabela do NCHS) demonstra a elevada capacidade do teste de Mann-Whitney na identificação de pe- quenas oscilações nos parâmetros antropométricos, mesmo em uma comunidade com pequeno número de habitantes.

Quando se avalia um mesmo grupo populacional em duas épocas distantes, pode-se observar variação na estatura como reflexo da tendência secular deste parâmetro antropométrico. Aumento da estatura associado à tendência secular vem sendo observada em várias populações do mundo, inclusive no Brasil (Kac, 1998; Monteiro et al., 1994). Observou-se aqui, redução no escore $Z$ da estatura para idade no período entre 1980 e 1992, ao contrário do observado na população brasileira e nas crianças índias Apache da América no Norte entre 1968 e 1988 (Hauck et al., 1992). Pode-se interpretar a diminuição ou a ausência de variação secular da estatura de duas maneiras: (1) deterioração das condições sócio-econômico-culturais de um grupo populacional resultante de condição ambiental inapropriada para o crescimento; ou (2) o grupo populacional em observação está vivendo em condições ideais e no passado já expressou com total plenitude seu potencial genético para o crescimento (Kac, 1999). Ao longo das últimas décadas, podem estar ocorrendo mudanças no padrão de vida dos índios do Alto Xingu e, neste contexto, a diminuição da estatura para a idade, mesmo limitada à faixa etária entre 1 e 5 anos, pode ser um sinal de alerta, indicando provável piora nas condições ambientais no Alto Xingu. É intrigante e sem explicação a caracterização de diminuição da estatura para a idade exclusivamente na faixa etária entre 1 e 5 anos. Em conclusão, nas crianças índias do Alto Xingu avaliadas na década de 1970, observou-se redução do déficit de estatura para a idade e do excesso de peso entre o primeiro e o quarto ano de vida. Por sua vez, entre 1980 e 1992, ocorreu diminuição da estatura para idade, indicando a importância da realização de novos inquéritos nutricionais com as crianças índias do Alto Xingu. 


\section{Referências}

ALVES, G. M. S.; MORAIS, M. B. \& FAGUNDES NETO, U., 2002. Estado nutricional e teste do hidrogênio no ar expirado com lactose e lactulose em crianças indígenas terenas. Jornal de Pediatria, 78:113119.

BARUZZI, R. G.; RODRIGUES, D. A.; WURKER, E.; AZEVEDO, R. A. \& MENDONÇA, S. B. M., 1990. Proposta para um Programa de Saúde. Atividades da Escola Paulista de Medicina em Colaboração com a Fundação Nacional do İndio. São Paulo: Escola Paulista de Medicina.

BLACK, F. L.; HIERHOLZER, W. J.; BLACK, D. P.; LAMM, S. H. \& LUCAS, L., 1977. Nutritional status of Brazilian Kayapo Indians. Human Biology Council, 49:139-153.

CAMPOS Jr., D., 2001. O crescimento de crianças indígenas. Jornal de Pediatria, 77:4-6.

FAGUNDES NETO, U.; BARUZZI, R. G.; WEHBA, J.; SILVESTRINI, W. S.; MORAIS, M. B. \& CAINELLI, M., 1981. Observations of the Alto Xingu Indians (central Brazil) with special reference to nutritional evaluation in children. American Journal of Clinical Nutrition, 34:2229-2235.

FRANCO, L., 1981. Aspectos Metabólicos da População Indígena do Alto Xingú (Brasil Central). Tese de Doutorado, São Paulo: Escola Paulista de Medicina.

GUGELMIN, S. A.; SANTOS, R. V. \& LEITE, M. S., 2001. Crescimento físico de crianças indígenas xavantes de 5 a 10 de idade em Mato Grosso. Jornal de Pediatria, 77:17-22.

HAUCK, F. R.; GALLAHER, M. M.; YANG-OSHIDA, M. \& SERDULA, M. K., 1992. Trends in anthropometric measurements among Mescalero Apache Indian preschool children: 1968 through 1988. American Journal of Diseases of Children, 146:11941198.

INAN (Instituto Nacional da Alimentação e Nutrição), 1990. Pesquisa Nacional sobre Saúde e Nutrição (Resultados Preliminares). Brasília: INAN.

JELLIFFE, D. B., 1968. Evaluación del Estado Nutricional de la Comunidad. Geneva: Organización Mundial de la Salud.

KAC, G., 1998. Tendência secular da estatura em recrutas da Marinha do Brasil nascidos entre $1940 \mathrm{e}$ 1965. Cadernos de Saúde Pública, 14:565-573.

KAC, G., 1999. Tendência secular em estatura: Uma revisão da literatura. Cadernos de Saúde Pública, 15:451-461.
MARTINS, S. J. \& MENEZES, R. C., 1994. Evolução do estado nutricional de crianças menores de 5 anos em aldeias indígenas da Tribo Parakana, na Amazônia Oriental Brasileira (1989-1991). Revista de Saúde Pública, 28:1-8.

MATTOS, A.; MORAIS, M. B.; RODRIGUES, D. A. \& BARUZZI, R. G., 1999. Nutritional status and dietary habits of Indian children from Alto Xingu (Central Brazil) according to age. Journal of the American College of Nutrition, 18:88-94.

MONTEIRO, C. A.; BENICIO, M. H. \& GOUVEIA, N. C. 1994. Secular growth trends in Brazil over three decades. Annals of Human Biology, 21:381-390.

MORAIS, M. B.; FAGUNDES NETO, U.; BARUZZI, R. G.; PRADO, M. C.; WEHBA, J. \& SILVESTRINI, W. S., 1990. Estado nutricional de crianças índias do Alto Xingu e avaliação do uso do perímetro braquial no diagnóstico da desnutrição protéicocalórica. Revista Paulista de Medicina, 108:245251

SANTOS, R. V., 1993. Crescimento físico e estado nutricional de populações indígenas brasileiras. Cadernos de Saúde Pública, 9:46-57.

SANTOS, R. V. \& COIMBRA Jr., C. E., 1991. Socioeconomic transition and physical growth of TupiMonde Amerindian children of the Aripuana Park, Brazilian Amazon. Human Biology, 63:795-819.

SIGULEM, D. M.; DEVINCENZI, U. M. \& LESSA, A. C. 2000. Diagnóstico do estado nutricional da criança e do adolescente. Jornal de Pediatria, 76:S275S284.

WATERLOW, J. C., 1972. Classification and definition of protein-calorie malnutrition. BMJ, 3:566-569.

WATERLOW, J. C.; BUZINA, R.; KELLER, W.; LANE, J. M.; NICHAMAN, M. Z. \& TANNER, J. M., 1977. The presentation and use of height and weight data for comparing the nutritional status of groups of children under the age of 10 years. Bulletin of the World Health Organization, 55:489-498.

WHO (World Health Organization), 1983. Measuring Change in Nutritional Status: Guidelines for Assessing the Nutritional Impact of Supplementary Feeding Programs for Vulnerable Groups. Geneva: WHO.

WHO (World Health Organization), 1995. Physical Status: The Use and Interpretation of Anthropometry. WHO Technical Report Series 854. Geneva: WHO.

Recebido em 18 de abril de 2002

Versão final reapresentada em 16 de outubro de 2002 Aprovado em 13 de dezembro de 2002 